\title{
Foreword
}

\section{The renin-angiotensin system and the heart}

One hundred years ago, Robert Tigerstedt and Per Bergman obtained extracts from the renal cortex of rabbits and discovered they had a pressor effect upon intravenous injection. Their paper, published 98 years ago, named the pressor substance "renin". It was not until the late 1930 s that it was proposed the renal pressor substance might be an enzyme. In 1958 the term "angiotensin" was coined for the active end product of the renin-angiotensin system (RAS).

Whereas most physiological research into the RAS system focused initially on its effects on blood vessels and arterial pressure, subsequent studies indicated that this system, through formation of angiotensin II, had diverse and widespread actions which affected functioning of the heart.

In so far as cardiac function is concerned, it is now clear that the RAS has numerous and complex effects. Circulating angiotensin II contributes to cardiac preload and afterload through its direct and indirect actions on blood vessels, and through its multiple effects on water and electrolyte balance. The inotropic, growth stimulating, and the toxic effects of angiotensin II may well play a pathophysiological role in clinical disorders such as acute myocardial infarction, essential hypertension, and congestive heart failure. Although attention has been focused on the kidney based circulating renin-angiotensin system, the existence of a separate, or at least partially independent, cardiac renin-angiotensin system, has received considerable attention in recent years.

The development of accurate methods for measuring plasma renin, and the ability of a small number of laboratories to measure circulating concentrations of angiotensin II accurately has been pivotal in our understanding of the RAS in so far as cardiac function is concerned. A further leap forward in defining the physiological and pathophysiological role of the RAS was the development of angiotensin converting enzyme (ACE) inhibitors. The therapeutic efficacy of ACE inhibitors in established heart failure, and after acute myocardial infarction for patients with impaired left ventricular systolic function, is now established. Their place in the drug treatment of patients with essential hypertension, and in renovascular hypertension, is debated. From a purely scientific view point, the ACE inhibitors are less than perfect in defining the role of the RAS, since they affect vasoactive systems in addition to the RAS. The subsequent development of selective angiotensin II receptor blocking agents, such as losartan, provide a further step forward to dissect the importance of the RAS system. Here again there will be limits to our understanding, since these blockers stimulate the release of renin from the kidneys, and while blocking the specific $\mathrm{AT}_{1}$ angiotensin II receptor, they leave other angiotensin II receptors available for stimulation, with uncertain biological consequences.

Notwithstanding scientific imperfections in our tools for dissecting the role of the RAS, there is no question that the cause of therapeutics in cardiological disorders has been dramatically advanced by the ACE inhibitors, and probably for the future, by angiotensin II receptor blockers.
The above issues are discussed by authors with a longstanding interest in various aspects of the RAS and the heart. The supplement begins, appropriately, with a historical background as we come towards the 100th anniversary of Tigerstedt's landmark publication. It will be apparent that there is now a considerable knowledge base which links cardiac structure and function with the RAS. Equally, there are areas of dispute such as the link between activity of the RAS and coronary artery disease.

What of the future? There will undoubtedly be advances in our understanding of the cardiac RAS. The current uncertainty as to whether genetic variation in components of the RAS, particularly relating to ACE activity, has a role in cardiac pathophysiology, needs to be clarified. It is both scientifically relevant and of practical therapeutic importance to know whether selective blockade of angiotensin II receptors has similar or different outcomes to ACE inhibition in heart failure, after myocardial infarction, and in essential hypertension. Whether the RAS indeed contributes to coronary atherogenesis is of scientific and therapeutic interest. Likewise, the role of the renin system in contributing to the development of left ventricular hypertrophy in essential hypertension requires better definition. As pointed out on numerous occasions and over many years by Framingham workers, the primacy of essential hypertension in the ultimate development of congestive heart failure needs to be remembered. In this context it is unclear whether agents which block the RAS have any advantage over alternative antihypertensive drugs in preventing the cardiac consequences of hypertension (heart failure in particular). It will be difficult to design studies capable of answering this question, since the trial drugs may need to be given under study conditions for a matter of decades in order to define accurately the comparative cardioprotective effects of agents which block, and those which stimulate, activity of the renin system.

The role of the RAS in so far as cardiac structure and function is concerned is complex and fascinating. The therapeutic implications of these complex interactions have been greater than anticipated. It is for these reasons that we look forward to the next decade when undoubtedly there will be improved understanding of how we can manipulate activity or actions of the RAS in the interest of maintaining cardiac function.

We thank the authors who contributed to this endeavour. Our referees have contributed significantly to the high standard of the manuscripts, for which we are grateful.

Finally, we wish to express our gratitude to Merck \& Co for sponsoring this supplement.

M GARY NICHOLLS Department of Medicine, Christchurch Hospital, Christchurch, New Zealand

HAMID IKRAM

Department of Cardiology, Christchurch Hospital, Christchurch, New Zealand 